\title{
PATENT CLASSIFICATION AS STIMULUS FOR INSPIRING NEW APPLICATIONS OF EXISTING KNOWLEDGE
}

\author{
Fiorineschi, Lorenzo; Frillici, Francesco Saverio; Rotini, Federico \\ University of Florence
}

\begin{abstract}
This paper aims to provide suggestions for the identification of potential new applications for the existing knowledge. A method is presented for extracting information about a product or technology, processing the international patent database (IPD) and extracting useful hints for potential new applications. The approach uses the Cooperative Patent Classification as stimulus for inspiring new potential fields towards which export existing product or technologies. Although some limits inevitably affect the approach, relevant directions for future developments have been inferred for a more comprehensive exploitation of both the firm internal knowledge and the suggestions provided by the international patent database. The achieved results can support firms in expanding market opportunities for their products or technologies.
\end{abstract}

Keywords: Innovation, Creativity, New product development

\section{Contact:}

Rotini, Federico

University of Florence

Industrial Engineering

Italy

federico.rotini@unifi.it 


\section{INTRODUCTION}

The satisfaction of customer needs leads companies to offer several sets of products focused on welldefined applications, but often neglect other potential uses of the developed knowledge and related technologies. Among the different causes behind this limited vision, psychological inertia (Savransky, 2000) plays a relevant role since it often leads people to unconsciously re-use the same personal paths. Indeed, years dedicated to develop and apply knowledge in specific industrial applications often lead designers and managers to blinker themselves to their own "safe solutions", neglecting new (but potentially risky) opportunities to successfully re-use their valuable knowledge in new industrial sectors. Even if several potential uses of an existing product are actually acknowledged for new application fields, fixation phenomena very often hinders attempts to notice these opportunities. To fill such a gap, some literature contributions propose to exploit the information provided by the International Patent Database (IPD) as creative stimuli to identify new opportunities. More precisely, information related to products or technology, already available to the firm, are used to perform patent searches for extracting potential stimuli. However, on the one hand, some of the mentioned contributions substantially fail in providing systematic and practical tools for a comprehensive and efficient exploitation of the resource, and in supporting objective analysis. On the other hand, other contributions currently requires to collect and pre-process a subset of the IPD, by exploiting ad-hoc developed software. In such a context, the authors developed a proposal aimed at overcoming the above-mentioned drawbacks (Fiorineschi et al., 2018). The method stimulates the user in identifying potential new applications of a product, by comprehensively exploiting the existing knowledge in the company or firm. It is implemented through elementary software tools and it is based on information retrieved from the Cooperative Patent Classification (CPC), which is used as a trigger to generate ideas for new product applications. During the last year, some case studies in different fields have been carried out, collecting encouraging results. The main objective of the paper is to show the problems emerged from the application of the mentioned approach, and to discuss about the related potential solutions. The content of the paper is structured as follows. Section 2 reports the background relevant for the topic, arising from the contributions available in literature. Section 3 briefly introduces the developed procedure and shows an illustrative application aimed at presenting the main outcomes of the approach. Discussions about limitations and problems of the procedure are summarized in Section 4, together with potential directions to solve these issues. Eventually, Section 5 reports the main conclusions.

\section{BACKGROUND}

The development of methods and approaches for identifying new opportunities constitutes an open research issue, where the patent database is often considered as a valuable resource. Accordingly, IPD is acknowledged as a valuable source of information for several innovation-related purposes (Akers, 2003). Currently, several patent analysis approaches exist (Abbas et al., 2014), and IPD is often exploited for technology forecasting purposes (e.g. Altuntas et al., 2015, Chen et al., 2017, Cho et al., 2017), i.e. for investigating technological trends and providing hints for future investments. However, another way exists to discover new opportunities, which is based on finding new applications to existing technologies and products (Yoon et al., 2015).

Among these contributions, some of them analyse the patents of the products owned by the firm to extract reliable suggestions and information for new product or technology development (Yoon et al., 2017). By exploiting different procedures, these approaches extract information from the descriptions of their products about firm internal capabilities, and then provide suggestions by referring to a partial subset of the IPD. Other proposals currently exists, which do not need to preliminary collect and preprocess a subset of patents, but they still refer to IPD for retrieving potentially useful suggestions for new applications of existing products or technologies. The first of these proposals is that suggested by Dewulf (2011), which proposes a method to link the examined products to other somewhat similar ones, to find new applications or new innovation possibilities throughout a comparison of product functions and properties. Verhaegen et al. (2011) proposes a method to find new applications based on similar products by considering a reference product and information gathered from patents. The similitude among products is evaluated by means of adjectives (e.g., hollow, smooth, flexible, etc.) present in product descriptions. Another proposal can be found in Gadd's book (2011) where the author asserts that inventions can be made not only by satisfying needs through the development of new solutions or new 
combinations of existing ones, but also by finding new uses or applications of extant systems, technologies, or functions. According to literature, IPD is therefore a potential useful resource of suggestions for new potential applications of products or technologies already available to the firm. The authors also support this evidence, but they believe that it is important also to provide systematic and practical tools for a better, agile and efficient exploitation of IPD to identify new application opportunities. The mentioned tools should also be capable to overcome psychological inertia problems and to perform more objective analysis. Accordingly, the following critical issues should be taken into account to develop suitable approaches based on IPD:

- When talking about functions it is necessary to consider that many different meanings can be associated with this term (Eckert et al., 2012; Vermaas and Eckert, 2013), often leading to misinterpretations and difficulties among people involved in the analysis.

- Product properties or keywords for formulating the search queries can be defined in a plethora of different ways, leading to high subjectivity of the achieved results.

- Processing only a part of the IPD could be limiting. However, since processing the entire IPD could be very cumbersome, it is necessary to find agile ways for extracting the stimuli for idea generation.

- Finally, to manage the process and to allow its repeatability, it is necessary to perform it systematically by following well-defined steps.

\section{USING CPC FOR IDENTIFYING NEW APPLICATION FIELDS}

The approach shown in the following paragraphs is claimed to overcome the issues that potentially hinder a comprehensive exploitation of the IPD, as introduced in the end of Section 2.

\subsection{Key concepts}

Before describing the proposed method, it is necessary to introduce the underpinning definitions. More in particular, since the method uses "functions" and "properties" to describe the "qualities" of the product, which might be relevant to transfer in other applications field, different interpretations of the same terms could lead to misunderstandings among people involved in the investigation/analysis process. Moreover, also an introduction to the CPC is provided.

\subsubsection{The cooperative patent classification}

The CPC is the emerging classification system that is going to become a standard in IPD, and can be used in many different patent search engines available online. It provides useful information about the application field of the invention. More precisely, the CPC is a hierarchical and alphanumeric classification of application fields, where some macro classes (e.g., Human Necessities, Chemistry and Metallurgy, Electricity, etc.) are coded with letters from A to Y. For each of the macro classes, an alphanumeric code of three or four symbols is used to identify first subclasses. For instance, in the A macro class, the A01 code refers to agricultural applications like agriculture itself, forestry, and animal husbandry; while the codes from A21 to A24 refer to foodstuff and tobacco-related applications, and codes from A61 to A63 refer to health and amusement. Similar subdivisions can be found for each of the mentioned macro classes. Furthermore, for most of the mentioned subclasses it is common to find other sublevels, where two sets of numeric codes are added to the initial codes, and are used to provide more detailed characteristics of the application field. For example, the CPC code "B03B 1/06" indicates that the invention is related to the "performing operations; transporting" macro class (B), then to "separating solid materials using liquids or using pneumatic tables or jigs" (B03B) subclass. Then, among the various typologies considered for the $\mathrm{B} 03 \mathrm{~B}$ subclass, the invention works by "conditioning for facilitating separation by altering properties of the matter to be treated" (B03B 1/00), more specifically "by varying ambient atmospheric pressure" (B03B 1/06).

\subsubsection{Product functions}

An industrial product can be considered a technical system, which delivers one or more functions according to a well-defined context of needs to be satisfied. Indeed, these functions and the way they are performed could be interesting knowledge for other application fields thus they should be correctly stated. Similarly to Yoon et al. (2015), among the different definitions of function that can be found in literature, for the scope of this work the authors considered the three different concepts of Subject (S), 
Action (A) and Object (O) according to Gadd (2011). More specifically, a function is considered as any action A performed by a subject $\mathrm{S}$, which somehow modifies the physical properties of an object $\mathrm{O}$ which receives A. Therefore, when a S-A-O triad is identified, the action of the triad represents a function performed by the subject. Besides the useful/desired actions performed by $\mathrm{S}$, it is convenient to consider also those harmful actions (HF) that $\mathrm{S}$ actually performs to the same $\mathrm{O}$ or other Os since those harmful actions can theoretically become useful actions if used in other contexts. For instance, a water jet can be used to cool a hot metal mold (water (S) cools (A) the mold $(\mathrm{O})$, useful function) but the pressurized water could enhance its erosion (water $(\mathrm{S})$ erodes $(\mathrm{HF})$ the mold $(\mathrm{O})$ ), which is a harmful function with respect to the considered context. However, the "erosion" action can be turned into something useful, for example, by substituting the object (thereby changing the application) with a "piece to be cut" by a highly pressurized water jet (i.e., the well-known water-cut of metal). Therefore, the triad becomes "water-jet (S)" "cuts (A)" a "metal slab (O)", where the erosion potentialities of the water-jet are now exploited to implement a useful function.

\subsubsection{Product properties}

The product owns specific features and characteristic, and delivers several performance that could be interesting also for new applications. Therefore, as well as functions, even these qualities represent a source for identifying new sectors where these features are relevant. It is difficult to formulate a comprehensive definition of properties and characteristics of a product, principally because these terms can be associated to an undefined set of meanings. For instance, a product can be small, big, beautiful, cumbersome, ergonomic, fast, slow, etc. depending principally on the product itself, but also on the type of evaluations that need to be performed. For technical applications, it is natural to focus on those physical properties and/or characteristics that can somehow be assessed by well-defined and objective metrics. Therefore, properties like speed, temperature, energy consumption, dimensions, etc. are considered here for the analysis of products. Nevertheless, subjective characteristics like aesthetic appearance, taste, comfort, etc. are also considered, because they actually characterize some products. For example, even if the perception of taste is subjective, everyone could agree that one of the most important characteristics of sugar is its sweet flavour.

\subsection{Description of the CPC-based approach}

The method for suggesting new product applications is articulated in the steps listed in Table 1 and described in the following paragraphs. It is based on patent search that is performed through specific queries. The terms used in the queries are defined according to the functions and the properties of the product, which are identified in reference to the definitions introduced in Section 4.1. The outcomes of the approach are specific CPC classes, which the user can employ as stimuli for identifying new knowledge applications.

Table 1: Framework of the CPC-based approach (Fiorineschi et al., 2018).

\begin{tabular}{|l|l|l|}
\hline Step & \multicolumn{1}{|c|}{ Description } & \multicolumn{1}{|c|}{ Expected outcomes } \\
\hline 1 & Eliciting input data from the firm staff & Information about the product \\
\hline 2 & Modelling of the product & Functions and properties of the product \\
\hline 3 & $\begin{array}{l}\text { Definition of the main keywords starting from the } \\
\text { functions and properties of the product }\end{array}$ & Main Keywords \\
\hline 4 & $\begin{array}{l}\text { Expansion of the main set of keywords into a more } \\
\text { comprehensive one }\end{array}$ & Expanded Keywords \\
\hline 5 & $\begin{array}{l}\text { Execution of a patent search using each Expanded } \\
\text { Keyword as a single query }\end{array}$ & $\begin{array}{l}\text { A set of patents for each Expanded } \\
\text { Keywords }\end{array}$ \\
\hline 6 & $\begin{array}{l}\text { Combinations of Expanded Keywords to define } \\
\text { multiple search queries }\end{array}$ & $\begin{array}{l}\text { Refined sets of patents according to the } \\
\text { combined queries }\end{array}$ \\
\hline 7 & Exportation and post-processing of the results & Refined set of CPC classes \\
\hline 8 & $\begin{array}{l}\text { Generation of ideas for new product applications } \\
\text { using the CPC classes }\end{array}$ & $\begin{array}{l}\text { A set of ideas for new possible } \\
\text { application of the product or part of it }\end{array}$ \\
\hline
\end{tabular}

Before starting with any search process, it is fundamental to understand what has to be searched (Step 1). In this case, it is necessary to elicit information about product functionalities and characteristics. In practice, several questions need to be asked to persons involved in the product development and 
commercialization, to gather as much detailed information as possible. To support this step, some well-known design checklists (Pahl et al., 2007; Pugh, 1991) can be used, where different groups of characteristics are listed to support the user in formulating comprehensive questionnaires and/or performing exhaustive interviews. Nevertheless, other checklists can also be found in literature, which can be used successfully for the stated purposes; e.g., that of Bacciotti et al. (2016).

Once the information from the performed interviews is retrieved, the data need to be analysed to extract the most representative functions and characteristics of the product; i.e., those that the interviewed staff acknowledges as the most important and/or peculiar (Step 2). This is not a structured process, but it is necessary to refer to a specific definition of function (e.g., that shown in 4.1.1) to avoid potential misunderstandings. Conversely, it is not possible to refer to standard definitions of properties and characteristics, but it is necessary to ensure that a comprehensive set of product descriptors is collected. In any event, for both functions and properties/characteristics, it is necessary to store not only those appearing as useful, but also those that the firm identifies as harmful.

Subsequently (Step 3), a meaningful set of keywords directly representative of the product should be defined, starting from the functions and the characteristics identified in the previous step. For instance, if the product is a pen, its main useful function is "marking a surface," while a harmful function is "to dirty fingers," and an important characteristic is that "it uses liquid ink". These are the most representative functions and characteristics of the product. Conversely, the consideration of secondary functionalities or characteristics (e.g., the possibility to have the pen in different colours) should be evaluated with care. Indeed, neglecting to consider secondary parameters could lead to an incomplete set of suggestions, but considering all the possible functions and properties of the product is likely to weigh down the entire process.

Unfortunately, by referring only to the initial list of keywords, many potentially relevant documents from the IPD could be not retrieved. Indeed, several synonyms can be found for many words, but also different forms of the same words can be present (e.g., generate, generated, generating), leading to incomplete search queries. Managing synonyms is a very important issue for the successful application of the proposed method (Step 4) since they represent different ways of expressing the same meaning. Commercial IPD search engines actually provide the option to use word truncations by inserting a single common root to reach all the different word formations (e.g., "generat"), allowing the composition of complex queries with Boolean operators to consider the various synonyms. A complex search query composed by different formulations and different synonyms of the same keyword can be called expanded keyword (EK). The operations can be performed manually, but also specific software platforms can be used to semi-automatically generate expanded keywords by starting with the initial set identified earlier. After the keyword expansion, a patent search in IPD search engines for each of the formulated EKs is carried out (Step 5). Patent search requires to indicate the fields to be inquired for performing the search. Initial searches should be carried out by referring only to the title (and eventually to the original title if present) and abstract of the patent documents, to avoid results characterized by high noise levels (i.e. a high number of not relevant documents). Indeed, if considering the description and/or the background fields, it is often likely to find the searched words but they could be inserted in secondary sentences not directly related to the main invention characteristics. Each set of results obtained for each EK will be tagged with a numeric code; for instance, in case of $\mathrm{n}$ search queries ( $\mathrm{n} E K s$ ), the $\mathrm{n}$ lists of patents are coded from 1 to $\mathrm{n}$.

Once the single searches have been conducted, intersections among the $n$ lists of patents are performed by combining the $n$ EKs through the Boolean operators (Step 6). Therefore, each combination of EKs expresses a sum of "concepts" that are available in a specific subset of patents. The maximum number of combinations $\mathrm{N}$ without repetitions is (Equation 1):

$$
N=\frac{n !}{k ! \cdot(n-k) !}
$$

where " $n$ " is the total number of patent lists while " $k$ " is the number of lists to be considered for the combinations. For example, in the case of 6 EKs $(n=6)$, the possible combinations can be performed by considering all the six retrieved lists of documents $(\mathrm{k}=6)$, then leading to a single possible combination $(\mathrm{N}=1)$. Again, considering groups of five lists $(\mathrm{k}=5)$, it is possible to obtain six possible combinations $(\mathrm{N}=6)$, and so on. Commonly commercial IPD search engines allow to automatically perform the requested intersections of the coded document lists. 
Once the relevant combinations have been defined, the proposed approach exports the CPC codes of the patents belonging to the subset of documents related to each combination (Step 7). More precisely, the first four symbols of the recalled CPC code are considered here; i.e., 660 sub-classes of the hierarchical CPC classification. This option is a good compromise between the most detailed information provided by a complete CPC code, and the need to retrieve a manageable set of information. Subsequently, the CPC classes potentially suitable for the examined product are identified according to the occurrences for each intersection of EKs. The obtained outcomes are the CPC classes, ordered from the most to the less recurring one, together with the related descriptions (indicating the application fields).

The Step 8 is the most subjective and creative one, because users should try to extract useful hints by using the identified CPC descriptions as a stimulus. In practice, since the identified CPC classes are associated by definition to particular applications, each of them indicates a potential new application field for the product. Therefore, the people involved in the investigation should ask themselves, "Is the highlighted application field suitable for our product?", "Does the considered CPC class suggest me something about a possible application of the product?".

\subsection{Illustrative application}

The case study briefly shown in this subsection aims at presenting the outcomes that can be obtained from the application of the approach. It refers to a grinding system for enhancing the production chain of the wood pellet. The system receives wood chips as input and provides sawdust as output. Thanks to rotating fan blades, the system pumps air and propels the wood chips. Air and wood chips are forced to pass through rotating and stationary blades that produce high frequency pressure waves that are partially adsorbed by the wood pieces. The impressed vibration and the numerous impacts are supposed to be the main effects that practically squeeze out micro-drops of water from wood, allowing it to reduce the moisture content to about $10 \%$. Unfortunately, the pressure waves also produce a high level of noise, which constitutes the main drawback of the system.

Table 2: Identified CPC classes for potential new applications (Fiorineschi et al. (2018))

\begin{tabular}{|c|c|c|}
\hline $\begin{array}{c}\text { EK } \\
\text { Combination }\end{array}$ & \begin{tabular}{|l|} 
CPC \\
Class
\end{tabular} & CPC class description \\
\hline \multirow[t]{2}{*}{$\begin{array}{l}\text { Grinding }+ \\
\text { dehumidify }+ \\
\text { pump }+ \\
\text { centrifugal }+ \\
\text { filter }\end{array}$} & $\mathrm{B} 02 \mathrm{C}$ & $\begin{array}{l}\text { Crushing, pulverising, or disintegrating in general; milling grain (household } \\
\text { tools and machines for pulverising foodstuffs, e.g. coffee and spice } \\
\text { mills A47J42/00; pharmaceutical mortars A61J3/02; mechanical processing } \\
\text { of refuse and garbage B03B9/06; dressing mould materials by } \\
\text { grinding B22C5/04; obtaining metallic powder by crushing, grinding or } \\
\text { milling B22F9/04; recovery of plastics by disintegrating B29B17/00; } \\
\text { crushing raw materials in starch making C08B30/02; beaters for } \\
\text { papermaking D21D1/02; crushing devices specially for transport in } \\
\text { mines E21F13/002; slag crushing devices F23J1/00; fuel milling devices in } \\
\text { combustion apparatus F23K1/00; household devices for crushing } \\
\text { coal F24B15/02; ice disintegrating devices F25C5/02) }\end{array}$ \\
\hline & $\mathrm{A} 23 \mathrm{~F}$ & $\begin{array}{l}\text { Coffee; tea; their substitutes; manufacture, preparation, or infusion } \\
\text { thereof (coffee or tea pots A47G19/14; tea infusers A47G19/16; apparatus } \\
\text { for making beverages, e.g. coffee or tea, A47J31/00; coffee mills A47J42/00) }\end{array}$ \\
\hline \multirow[t]{2}{*}{$\begin{array}{l}\text { Grinding }+ \\
\text { dehumidify }+ \\
\text { pump }+ \\
\text { centrifugal }\end{array}$} & A23L & $\begin{array}{l}\text { foods, foodstuffs, or non-alcoholic beverages, not covered by } \\
\text { subclasses a } 23 \mathrm{~b} \text { - a23j; their preparation or treatment, e.g. cooking, } \\
\text { modification of nutritive qualities, physical treatment (shaping or working, } \\
\text { not fully covered by this subclass, a23p); preservation of foods or foodstuffs, } \\
\text { in general }\end{array}$ \\
\hline & $\mathrm{C} 10 \mathrm{~F}$ & drying or working-up of peat \\
\hline
\end{tabular}

The main functions and properties of the system have been extracted and used to define the main keywords according to Steps 1, 2 and 3 of Table 1. Subsequently, each main keyword has been expanded and a specific set of EKs has been defined according to Step 4 (further details available at this link https://goo.gl/FNhtJ4). Hence, each EK has been used for performing a patent search with the Orbit software (Orbit, 2017), by focusing the search on title, original title, and abstract of all active documents (Step 5). Afterwards, EKs have been combined according to the Equation 1 (Step 6) and 
the results have been managed and exported in the form of the CPC classes (Step 7). After this process, a suitable set of CPC classes has been highlighted (Table 2) from which to extract potentially useful hints (Step 8).

For instance, the classes B02C and A23F suggested to think about the grinding of coffee or tea leaves, while the A23L led to the grinding and dehumidification of edible materials. Similarly, the A23G suggested the grinding and dehumidification of cocoa seeds, while the $\mathrm{C} 10 \mathrm{~F}$ class led to think about the grinding of carbon and peat. Indeed, since no firm and/or expert has been involved in the process, the authors did not collect feedback about the feasibility and the utility of the considered grinding system within the context of the individuated new applications.

Nevertheless, the case study demonstrated that the considered approach is capable to provide stimulation means in form of examples, for triggering the creative thinking, which was the main expected performance of the method. However, the authors are conscious that the collected results are not sufficient for a full and comprehensive validation of the proposal. Indeed, the conditions under which the provided examples can actually overcome or induce fixation are not clear.

\section{PROBLEMS OF THE APPROACH AND POTENTIAL SOLUTIONS}

The development of a comprehensive software platform for an agile application of the approach shown in Section 4 is certainly a desirable target but, before doing that, some fundamental issues need to be faced about certain aspects.

\subsection{Tailored checklists}

The checklists used for the first step of the approach (i.e., eliciting information from firm staff) were originally developed for different purposes, so it could be preferable to develop a more effective and detailed version for the present aims and scopes. More specifically, it is important to provide structured instructions about what kind of information is needed, and about the form in which product information should be provided (e.g. textual description, textual and pictorial description, etc.). Indeed, different ways to provide initial description could differently affect fixation or psychological inertia (Vasconcelos et al., 2017), and then differently affect the extraction of the keywords (Step 3 in Table 1). The adaptation to the task of the contribution presented in (Bacciotti et al., 2016) could represent a possible solution for a more systematic identification and description of product properties and characteristics. The mentioned contribution refers to a structured approach for investigating the product features according to two different dimensions, i.e. characteristics relevant for different stakeholders and in different phases of the product life cycle. The approach has been implemented through a software named "IDEA", which generates questions according to the dimensions the user wants to investigate. Therefore, the analysis of the product properties is conducted by using a structured path, which leads to a comprehensive and exhaustive analysis.

\subsection{Decomposition models}

The identification of functions and properties should be supported more systematically. To this purpose, some design tools can be used for a comprehensive product decomposition, like function trees (Malmqvist, 1997; Robotham, 2002), problem and solution networks (Fiorineschi et al., 2016) or function structures (Figure 1). Moreover, available functional basis (Hirts et al., 2002; Stone and Wood, 2000) could be exploited to allow a standardized formulation of functions, as well as automatic natural language processing procedures (Cascini et al., 2004; Yoon et al., 2015). However, some research activity is still needed to identify the most suitable model to be considered for the purposes of the present approach. In particular, real case studies should be considered in future activities, where companies and/or experts are directly involved in the experiments.

\subsection{Supporting the identification of the most suitable EKs combinations}

The identification of preferred EK combinations should be supported in a more structured way, for example, by means of systematic guidelines providing indications for focused and iterative information gathering activities. Similarly, for what concerns the identification of the preferred CPC classes, we observed that, by referring only to the most frequently recurring ones, it is possible to obtain an incomplete set of suggestions. Actually, as observed by Singh and Terzidis (2015), some correlations can be observed between patent filings and product counts, which could provide additional information 
for supporting the identification of preferred CPCs. Nevertheless, it has also been observed that companies may pursue different patent portfolio strategies (Chang and Chang, 2016), referring to CPC patent counts not being sufficient for identifying preferred application fields. Therefore, it is necessary to understand if some correlations exist between the number of CPC occurrences and the value of the suggestions provided by the related description. It could be done by applying the process to several case studies, where the goodness of the proposed new product application is assessed, for example, by well known evaluation matrices (Akao, 1990; Pugh, 1991) where evaluation parameters are selected with the firm staff. However, this empirical research strategy could result onerous for the definition of suitable criteria to select and prioritize the most suitable combinations among EKs. This aspect of the developed approach is the most critical and the research is still open, therefore the authors are open to any useful suggestion about the topic. Accordingly, Gonçalves et al. (2016) highlighted the importance of defining keywords when performing search processes. The same authors also observed that it is still an open research issue.

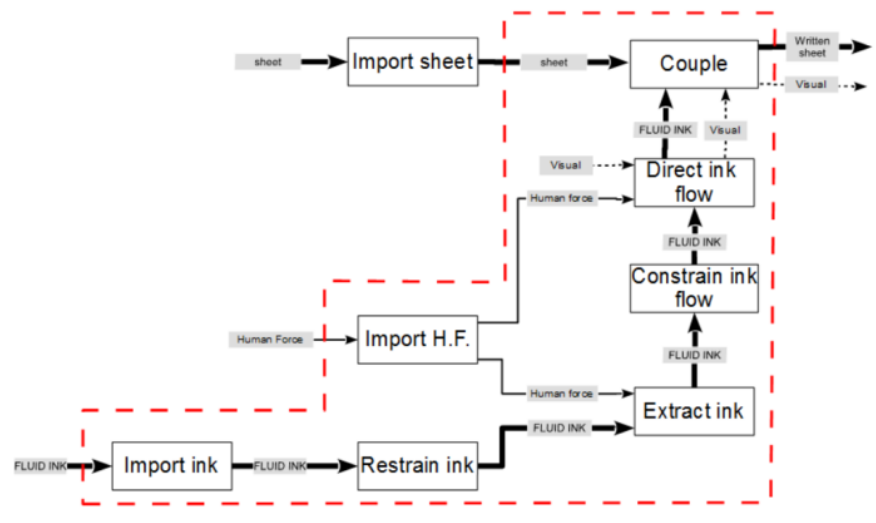

Figure 1. Functional model of a pen (from Cascini et al. (2018)).

\subsection{Idea generation step}

The idea generation step (i.e., the last step of the process) should be supported more systematically to guide the users through a detailed ideation path, where the identified CPC classes act as creative stimuli. Design literature acknowledges several studies about the effect of stimuli in idea generation (Chiu and Shu, 2012; Goldschmidt and Sever, 2011; Gonçalves et al., 2014; Howard et al., 2010; López-Mesa et al., 2011), which, however, are not directly applicable to the present purposes. Nevertheless, existing literature could certainly provide useful hints for future research activities devoted to understanding the effect of the textual stimuli provided by CPC classes on the generation of new ideas for product applications. Moreover, it would be possible to extract suitable research queries from the CPC classes, to be used for image searches to be performed on the web. For example, considering the CPC classes identified in Table 2, and referring to the A23F, the following research query can be extracted: "Coffee manufacture". By performing an image search with the Google engine, many images recalling the need of "grinding" the coffee can be easily found (Figure 2). This could better support the idea generation process (e.g. could the same grinding technology be applied to the coffee manufacturing process?). However, the actual correlation between the images extracted from web search engines and the contents of the specific CPC code is unclear. Consequently, more research is needed to evaluate the applicability of this solution to the approach described in this paper.
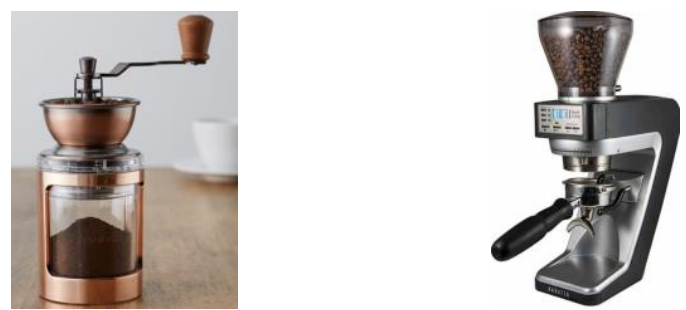

Figure 2. Coffee grinder images from web searches performed with the query "Coffee manufacture", extracted from the A23F CPC class. 
Anyway, it is necessary to assess the actual impact of the approach in terms of creativity of the generated "application" ideas. Accordingly, opportune creativity metrics should be identified and/or developed in future activities. Indeed, the applicability of classical creativity metrics, normally used to assess design concepts and ideas, should be verified for the outcomes of this specific approach (where the term "application" is intended as "destination of use").

\section{CONCLUSIONS}

The paper discusses an approach to support the identification of new applications of existing firm's knowledge by using CPC classes as stimulus. Furthermore, it presents the main problems that still affect the proposed procedure, and discusses potential directions to solve them. If compared with the contributions available in literature (see Section 2), some specific features characterize the CPC-based approach described in Section 3. In reference to the semi-automated approaches proposed by Park and Yoon (2017), Seo et al. (2016), Yoon et al. (2015, 2017), the considered approach does not need to collect and pre-process any subset of the IPD. Indeed, the procedure requires product information directly extracted from the firm staff by means of interviews and questionnaires, and it directly refer to CPC. Therefore, the considered approach can be applied also to products or technologies that are already available to the firm but not necessarily patented. Moreover, the procedure currently does not use a specific software or a specific repository of patents, and then everyone can apply it in its current form. Furthermore, since the considered approach processes the entire IPD, it allows a more comprehensive exploitation of such a resource, and then could potentially provide a more extended set of suggestions.

Eventually, future improvements could certainly lead to automated and optimized procedures, also allowing the development of a user-friendly software platform. However, before doing that, testing activities are needed where companies and/or experts are directly involved. Indeed, future activities should be focused on both the identification of the criticalities of the approach from real applications, and the assessment of the actual impact of the generated ideas (e.g. in terms of creativity). Nevertheless, it is possible to assert that the approach shown in this paper already constitutes a new resource for innovation consultants and firms, as well as for other subjects involved in similar studies. Further future developments of the procedure will concerns the investigation of the potential solutions discussed in Section 4.

\section{REFERENCES}

Abbas, A., Zhang, L. and Khan, S.U. (2014), "A literature review on the state-of-the-art in patent analysis", World Patent Information, Elsevier Ltd, Vol. 37, pp. 3-13.

Akers, L. (2003), "The future of patent information - A user with a view", World Patent Information, Vol. 25 No. 4, pp. 303-312.

Altuntas, S., Dereli, T. and Kusiak, A. (2015), "Forecasting technology success based on patent data", Technological Forecasting and Social Change, Elsevier Inc., Vol. 96, pp. 202-214.

Bacciotti, D., Borgianni, Y. and Rotini, F. (2016), "Computers in Industry An original design approach for stimulating the ideation of new product features", Computers in Industry, Elsevier B.V., Vol. 75, pp. 80-100.

Cascini, G., Fantechi, A. and Spinicci, E. (2004), "Natural language processing of patents and technical documentation”, Document Analysis Systems VI, pp. 508-520.

Cascini, G., Fiorineschi, L. and Rotini, F. (2018), "Investigating on the Re-use of Conceptual Design Representations", International Design Conference - Design 2018, pp. 1009-1020.

Chang, S.-H. and Chang, H.-Y. (2016), "The study of patent portfolio strategies of oil shale developers", International Journal of Innovation Science, Vol. 8 No. 3, pp. 254-268.

Chen, H., Zhang, G., Zhu, D. and Lu, J. (2017), “Topic-based technological forecasting based on patent data: A case study of Australian patents from 2000 to 2014", Technological Forecasting and Social Change, Elsevier Inc, Vol. 119, pp. 39-52.

Chiu, I. and Shu, L.H.H. (2012), "Investigating effects of oppositely related semantic stimuli on design concept creativity”, Journal of Engineering Design, Vol. 23 No. 4, pp. 271-296.

Cho, H.P., Lim, H., Lee, D., Cho, H. and Kang, K.I. (2017), "Patent analysis for forecasting promising technology in high-rise building construction", Technological Forecasting and Social Change, Elsevier, No. September 2016, pp. 0-1.

Dewulf, S. (2011), "Directed variation of properties for new or improved function product DNA - A base for connect and develop", Procedia Engineering, Elsevier B.V., Vol. 9, pp. 646-652. 
Eckert, C., Ruckpaul, A., Alink, T. and Albers, A. (2012), "Variations in functional decomposition for an existing product: Experimental results", Artificial Intelligence for Engineering Design, Analysis and Manufacturing, Vol. 26 No. 2, pp. 107-128.

Fiorineschi, L., Rotini, F. and Rissone, P. (2016), "A new conceptual design approach for overcoming the flaws of functional decomposition and morphology”, Journal of Engineering Design, Vol. 27 No. 7, pp. 438-468.

Fiorineschi, L., Frillici, F.S., Gregori, G. and Rotini, F. (2018), "Stimulating idea generation for new product applications", International Journal of Innovation Science, Vol. 10 No. 4, pp. 454-474.

Gadd, K. (2011), TRIZ for Engineers: Enabling Inventive Problem Solving, John Wiley and sons, Inc, available at: https://doi.org/10.1002/9780470684320.

Goldschmidt, G. and Sever, A.L. (2011), "Inspiring design ideas with texts”, Design Studies, Elsevier Ltd, Vol. 32 No. 2, pp. 139-155.

Gonçalves, M., Cardoso, C. and Badke-Schaub, P. (2014), "What inspires designers? Preferences on inspirational approaches during idea generation", Design Studies, Vol. 35 No. 1, pp. 29-53.

Gonçalves, M., Cardoso, C. and Badke-Schaub, P. (2016), "Inspiration choices that matter: the selection of external stimuli during ideation”, Design Science, Vol. 2 No. E10, pp. 1-31.

Hirts, J., Stone, R.B., McAdams, D.A., Szykman, S. and Wood, K.L. (2002), “A Functional Basis for Engineering Design: Reconciling and Evolving Previous Efforts”, National Institute of Standards and Technology.

Howard, T.J., Dekoninck, E.A. and Culley, S.J. (2010), “The use of creative stimuli at early stages of industrial product innovation", Research in Engineering Design, Vol. 21 No. 4, pp. 263-274.

López-Mesa, B., Mulet, E., Vidal, R. and Thompson, G. (2011), "Effects of additional stimuli on idea-finding in design teams", Journal of Engineering Design, Vol. 22 No. 1, available at: https://doi.org/http://dx.doi.org/10.1080/09544820902911366.

Malmqvist, J. (1997), “Improved Function-Means Trees by Inclusion of Design History Information”, Journal of Engineering Design, Vol. 8 No. 2.

"Orbit". (2017), https://www.orbit.com/.

Pahl, G., Beitz, W., Feldhusen, J. and Grote, K.H. (2007), Engineering Design 3rd Ed, Springer-Verlag, London.

Park, Y. and Yoon, J. (2017), “Application technology opportunity discovery from technology portfolios: Use of patent classification and collaborative filtering", Technological Forecasting and Social Change, Elsevier Inc., Vol. 118, pp. 170-183.

Pugh, S. (1991), Total Design. Integrated Methods for Succesfull Product Engineering, Addison Wesley Publishing Company, Reading, Massachusetts.

Robotham, A.J. (2002), “The use of function/means trees for modelling technical, semantic and business functions", Journal of Engineering Design, pp. 243-251.

Ross, S.M. (2014), Introduction to Probability and Statistics for Engineers and Scientists, Academic Press.

Savransky, S.D. (2000), Engineering of Creativity: Introduction to TRIZ Methodology of Inventive Problem Solving, CRC Press.

Seo, W., Yoon, J., Park, H., Coh, B., Lee, J.-M. and Kwon, O.-J. (2016), "Product opportunity identification based on internal capabilities using text mining and association rule mining", Technological Forecasting and Social Change, Elsevier Inc., Vol. 105, pp. 94-104.

Singh, M.D. and Terzidis, O. (2015), “Introducing Innovation Phase Transition”, International Journal of Innovation Science, Vol. 7 No. 4.

Stone, R. and Wood, K. (2000), "Development of a Functional Basis for Design”, Journal of Mechanical Design, Vol. 122 No. 4, pp. 359-370.

Vasconcelos, L.A., Cardoso, C.C., Maria, S., Chen, C. and Crilly, N. (2017), "Inspiration and Fixation : The Influences of Example Designs and System Properties in Idea Generation", Journal of Mechanical Design, Vol. 139 No. March, pp. 1-13.

Vermaas, P.E. and Eckert, C. (2013), "My functional description is better!", Artificial Intelligence for Engineering Design, Analysis and Manufacturing, Vol. 27 No. 3, pp. 187-190.

Yoon, J., Park, H., Seo, W., Lee, J.M., Coh, B.y. and Kim, J. (2015), “Technology opportunity discovery (TOD) from existing technologies and products: A function-based TOD framework", Technological Forecasting and Social Change, Elsevier Inc., Vol. 100, pp. 153-167.

Yoon, J., Seo, W., Coh, B.Y., Song, I. and Lee, J.M. (2017), "Identifying product opportunities using collaborative filtering-based patent analysis", Computers and Industrial Engineering, Elsevier Ltd, Vol. 107, pp. 376-387.

\section{ACKNOWLEDGMENTS}

The authors acknowledge the contribution of "Fondazione Cassa di Risparmio di Pistoia e Pescia", which partially supported this research through the funding programme named "Bando Giovani e Ricerca 2016". 